\title{
Trajectories of Accumulation of Health Deficits in Older Adults: Are There Variations According to Health Domains?
}

Esther García-Esquinas MD, $\mathrm{PhD}$ a,b, ${ }^{*}$, Rosario Ortolá a, Matthew Prina $\mathrm{PhD}$ c, Denes Stefler PhD, MSc d, Fernando Rodríguez-Artalejo MD, PhD a,b, Roberto Pastor-Barriuso PhD, MSc b,e

\footnotetext{
a Department of Preventive Medicine and Public Health, Universidad Autónoma de Madrid and Idipaz, Madrid, Spain b CIBER of Epidemiology and Public Health (CIBERESP), Madrid, Spain

c Department of Health Service and Population Research, Institute of Psychiatry, Psychology \& Neuroscience, King's College London, United Kingdom

d Department of Epidemiology and Public Health, University College London, United Kingdom

e National Center for Epidemiology, Carlos III Institute of Health, Madrid, Spain
} 


\section{Abstract}

2 Objectives: To analyze sex- and education-specific trajectories of health deficits

3 accumulation (DA) throughout old age, and to examine whether these trajectories differ

4 according to health domains.

5 Design: Population-based prospective cohort study

6 Setting and participants: In Spain, 3,228 community-dwelling individuals aged 60

7 years or older followed-up for 10 years.

8 Measures: At baseline and three follow-up visits, a DA index (0 to $100 \%$ deficit) was

9 calculated based on the number and severity of 52 health deficits across the domains of physical and cognitive function, self-rated health and vitality, mental health, and morbidity. Trajectories of overall and domain-specific DA indexes were estimated using mixed models for repeated measurements, allowing for homogeneous linear, piecewise linear and smooth nonlinear DA trends over age.

14 Results: Women showed greater DA than men, but differences leveled out with age: annual increments in DA index (95\% confidence interval) for participants aged $<70,70$ 80 and $\geq 80$ years were, respectively, $0.40(0.30-0.50), 0.68(0.57-0.80)$, and $1.30(1.07$ $1.54)$ in men; and $0.63(0.53-0.73), 0.99(0.89-1.10)$ and $1.31(1.09-1.53)$ in women. These changes were mainly driven by a progressive accumulation of chronic diseases and by quadratic declines in physical and cognitive function. Both men and women showed improvements in mental health with age. In general, women and participants with lower education decreased physical and cognitive function and accumulated morbidities faster than their counterparts. Only individuals with very fast declines in 
23 function and fast accumulation of morbidities showed a significant worsening of self-

24 rated health and vitality over time.

25 Conclusions: Public health interventions aimed to healthy ageing should focus on

26 prevention and early-control of chronic diseases, as well on the preservation of function

27 and early rehabilitation, with the latter being more relevant during the latter stages of

28 life. Post-acute and long-term care services should incorporate adequate physical

29 therapy and nursing facilities to promote attainable levels of functionality in older

30 adults.

31 Key words: Deficit accumulation, function, healthy ageing, mental health, mixed

32 models, morbidity, repeated measures

33 
35 Ageing is the result of cumulative molecular and cellular damage over time, which leads to a progressive decline in physical and cognitive function, and a growing risk of chronic diseases, disability and death [1]. Ageing does not evolve linearly throughout old age and greatly differs across subjects. Monitoring ageing pathways or trajectories in older adults is needed to understand how these changes unfold and helps identify which public health interventions may be effective at a given time to prevent, treat or support the decline in specific abilities with age [2].

42 Over the past years, an increasing number of longitudinal studies have evaluated trajectories of health deficits accumulation (DA) in older adults and their main determinants [3-9]. Most of these studies have relied on the frailty index, a multidimensional index defined as the proportion of health deficits present in an individual out of the total number of deficits considered, which has shown to be a good

47 predictor of adverse outcomes (i.e., falls, hospitalization, disability, institutionalization and death) in older adults [10]. However, none of these studies has specifically evaluated DA trajectories across the different health domains that encompass this syndrome, such as physical function, mental health and morbidity. Within this context, the present manuscript aims to deepen understanding of the dynamics of ageing by examining the overall and domain-specific DA trajectories over age in the 10-year follow-up of a representative cohort of 3,289 community-dwelling older adults in Spain.

\section{METHODS}

\section{Study population}


The Seniors-ENRICA cohort was established in 2008-2010 with 3,289 individuals selected by multi-stage stratified random sampling from the non-institutionalized Spanish population aged 60 years or older [11;12]. Of the initial cohort, 61 participants were excluded from the present study because they lacked information on health deficits or potential confounders. The remaining 3,228 participants were followed-up until 2017 , for a total of 8,562 baseline and follow-up visits (mean follow-up time between successive visits of 2.5 years). During the 10 -year study period, 267 participants $(8.3 \%)$ died, 1,840 (57.0\%) were lost to follow-up due to non-response, and the remaining 1,121 (34.7\%) completed the three follow-up visits (supplementary figure 1). Participants lost to follow-up were more likely to be women $(59.1 \%$ vs $51.0 \%$; $\mathrm{p}<0.001)$, older (69.1 vs 67.1 years; $\mathrm{p}<0.001)$ and had lower education level $(62.3 \%$ with primary education or less vs $48.0 \%$; $\mathrm{p}<0.001$ ) than those who took part in all follow-up visits.

Baseline and follow-up information regarding sociodemographic, lifestyle, self-rated health and morbidity was collected using computer-assisted telephone interviews and dietary histories. Samples of urine and blood were collected and mental and physical exams were performed by trained staff in home visits. All participants gave informed consent, and the Clinical Research Ethics Committee approved the study $[11 ; 12]$.

\section{Health deficits accumulation and lifestyle risk factors}

Based on the Rockwood's frailty index [10], a DA index was calculated at baseline and each follow-up visit using a total of 52 health deficits, including 22 impairments in physical and cognitive functioning, 7 self-reported health and vitality problems, 6 mental health deficiencies and 17 items on morbidities, polypharmacy and use of health services. The index summarizes age-related vulnerability, so the more health deficits 
(symptoms, signs, diseases or disabilities) an individual has, the higher their risk of death, institutionalization, health service use or further deficit accumulation. Most deficits in the index were assessed in a dichotomous way (1 point if present and 0 otherwise), with the exception of cognitive functioning, self-rated health, vitality, mental health, body mass index and use of outpatient health care, whose deficits were graded according to severity ( 0 points for no deficit, 0.25 to 0.75 points for mild to moderate deficits and 1 point for severe deficit). The complete list of health deficits and associated scores are outlined in supplementary table $\mathbf{1}$ and a detailed description of the construction of frailty index domains is provided in Methodological Appendix 1.

The overall DA score was calculated as the sum of points assigned to each health deficit divided by the 52 deficits considered and further multiplied by 100 to obtain a summary range from 0 to $100 \%$ deficit.

At baseline and follow-up visits, self-reported information was obtained on age, sex, educational level, smoking status and alcohol consumption. Physical activity was measured with the questionnaire used in the EPIC-Spain cohort [13], and sedentary behavior was estimated by time spent watching television as assessed by the Nurse's Health Study questionnaire validated in Spain [14]. There is evidence that time spent watching TV is the main component of sedentary time among older adults [15-17], and, compared to other types of sedentary behaviors, shows a distinct demographic and health profile [18]. Food consumption in the previous year was collected using a validated computerized dietary history developed from that used in the EPIC-Spain cohort [19]. Adherence to the Mediterranean diet was summarized with the Mediterranean Diet Adherence Screener index [20]. Height and weight were measured on physical exams in home visits and body mass index was calculated as weight in kilograms divided by height in meters squared. 


\section{Statistical methods}

The longitudinal change in the DA index with age was estimated by using linear mixed models for repeated measurements over time on the same participants $[21 ; 22]$. The models are specified in detail in the Methodological Appendix 2. In brief, a linear mixed model with random intercept and random age slope was used to estimate the average longitudinal change in DA index per one-year increase in age, while allowing for individual variation in DA trajectories around the average linear trend. This mixed model was then extended with fixed coefficients for linear splines of age with knots at 70 and 80 years in order to estimate distinct longitudinal annual changes in DA index within age intervals $60-69,70-79$, and $\geq 80$ years, as well as with fixed coefficients for restricted quadratic splines of age with identical knots in order to display the smooth longitudinal trend in DA index with age [23].

To control for potential sociodemographic confounders and to assess the longitudinal effect of age on DA index mediated by lifestyle changes, the above mixed models were fitted with (models II-VI) and without (model I) adjustment for sociodemographic and lifestyle risk factors, including fixed effects for sex (men or women), educational level (primary or less, secondary, or university), baseline Mediterranean diet score (0 to 14 points), and changes over time in smoking status (never, former, or current), alcohol drinking (never, former, moderate, or heavy), physical activity (MET-hours/week), sedentary behavior (TV hours/day), and body mass index $\left(\mathrm{kg} / \mathrm{m}^{2}\right)$. To evaluate potential heterogeneity of DA trajectories by sex and education, interactions of sex or educational level with the linear age term, linear age splines, and restricted quadratic age splines were included as fixed effects in the corresponding mixed models. 
128 In addition to the overall DA index, mixed models were also used to estimate

129 longitudinal changes in domain-specific DA indexes (physical and cognitive function,

130 self-rated health and vitality, mental health, and morbidity) adjusting for changes over

131 time in the other health dimensions. Modifications in domain-specific DA trajectories

132 by quartile of change in other dimensions were evaluated including fixed-effects

133 interactions between these quartiles and the abovementioned age terms. Statistical

134 analyses were performed in Stata, version 14 (StataCorp) and graphics were produced in

$135 \mathrm{R}$, version 3 (R Foundation for Statistical Computing).

\section{RESULTS}

Participants mean age at baseline was 69.0 years (range 6.6). There was a higher proportion of women $(53.8 \%)$ than men $(46.2 \%)$. More than half of the study

participants only had primary education or less and had never smoked, an approximately one third had never drunk, all these proportions being higher among women. Men showed a higher adherence to the Mediterranean dietary pattern, were more physically active, watched less TV, and had lower mean DA index scores than women at baseline. Participants with higher education were more frequently men, younger, and more active, and showed a lower prevalence of smoking and obesity , a higher prevalence of alcohol consumption and a better adherence to the Mediterranean dietary pattern. (Table 1).

The baseline mean (range) number of health deficits was 2.7 (0-22) for physical and cognitive function, 4.2 (0-7), for self-rated health and vitality, 1.2 (0-6) for mental health, and 3.6 (0-17) for morbidities. Compared to participants in the lowest quartile of baseline DA index, as well as in the functional impairments, self-rated health and vitality, and morbidities domains, those in the highest quartile exhibited an increased 
death risk during the 10-year follow-up: hazard ratios (95\% confidence intervals [CI]) were $2.87(1.87,4.42), 1.95(1.29,2.94), 1.77(1.25,2.52)$ and $1.67(1.13,2.46)$, respectively (data not presented in tables).

Table 2 shows the longitudinal changes in overall and domain-specific DA indexes per one-year increase in age and their 95\% CI. In crude models (model I), on average, participants accumulated 0.40 deficits per year, with an accelerated increase with age. These 0.40 deficits correspond to the 0.77 percentage annual change in the DA index observed in model I. Because the DA index ranges from 0 to 52 , a $0.77 \%$ change in the DA scale would correspond to an accumulation of 0.40 deficits $[0.40=0.77$ unadjusted annual change in overall DA index] $* 52_{\text {[maximum number of health deficits] }}(100)$ ). These figures remained almost unchanged after adjustment for baseline educational level and changes over time in health behaviors. Results from interaction models showed that women aged 60-79 had greater DA than men, but differences leveled out with age; specifically, at ages 60-69, 70-79 and $\geq 80$, average increments in the DA index were (model II), respectively, 0.40 $(0.30,0.50), 0.68(0.57,0.80)$ and $1.30(1.07,1.54)$ in men; and $0.63(0.53,0.73), 0.99$ $(0.89,1.10)$ and $1.31(1.09,1.53)$ in women. Both men and women increased functional loss [mean annual increases in the deficits score over the entire follow-up (95\% CI): 0.90 $(0.76,1.04)$ and $1.40(1.26,1.54)$, respectively; model III], experienced no changes in self-rated health and vitality $[0.04(-0.08,0.16)$ and $-0.03(-0.15,0.09)$, respectively; model IV], had progressive improvements in mental health $[-0.97(-1.13,-0.81)$ and $1.17(-1.33,-1.01)$; model V], and underwent an increase in their number of chronic conditions and health care visits $[0.79(0.72,0.86)$ and $0.93(0.86,1.00)$; model VI] with age. In general, women lost function, improved mental health and accumulated morbidities faster than men (p-values for interaction were $<0.001,0.06$ and $<0.001$, respectively). Figure 1 shows the smooth longitudinal trend in the overall DA index, 
and helps illustrating both the accelerated increase in the frailty index with age, as well as the observed sex differences. Additionally, this figure shows that DA index changes are driven both by increasing morbidities and function decline, with the latter being more relevant in older age. Moreover, it shows that women bear a larger burden of health deficits at any given time and in all dimensions.

Table 3 and Supplementary figure 2 present changes in the overall DA index and its main dimensions according to baseline educational status. In general, participants in the highest educational level exhibited the lowest scores in the studied domains at all ages (p-values for homogeneity of annual changes among participants with different educational level were $<0.001$ for the overall DA index, $<0.001$ for the functional impairments dimension, 0.44 for the self-rated health and vitality dimension, $<0.001$ for mental health, and 0.02 for morbidities). However, differences were not statistically significant after the age of 70 (p-values for homogeneity of annual DA changes among participants with different educational level 0.49 and 0.48 for those aged $70-79$ and $\geq 80$, respectively).

Finally, figure 2 exhibits changes in each DA index dimension according to quartiles of change in the other three. On average, participants who experienced the highest declines in self-rated health and vitality, the lowest improvements in mental health, and the fastest accumulation of chronic diseases, presented the fastest declines in function [mean changes for those in the fourth vs first quartile of self-rated, mental, and chronic health changes: 2.55 (2.34 to 2.75$), 1.71$ (1.51 to 1.91$)$, and 1.63 (1.44 to 1.81 ), respectively]. Similarly, participants who suffered the fastest deterioration in function or the fastest accumulation of morbidities, showed the sharpest declines in self-perceived health and vitality [mean annual change for those in the fourth vs first quartile of 
201 functional deterioration and morbidities accumulation: 0.92 (0.75 to 1.10) and 0.38

202 (0.22 to 0.54), respectively]; while those who suffered the fastest deterioration in

203 functional, self-rated, and mental health, displayed the fastest increments in number of

204 chronic diseases [mean annual increase in the number of chronic conditions for those in

205 the fourth vs first quartile of functional deterioration, self-rated health decline, and

206 mental health improvement: 1.23 (1.13 to 1.33$), 1.34$ (1.24 to 1.45$)$, and 1.04 (0.94-

207 1.14), respectively].

208 As sensitivity analyses, we repeated all models including only participants with at least

209 three follow-up visits, with consistent findings (see supplementary table 2).

\section{DISCUSSION}

211 Our results provide important information on the dynamics of ageing. First, they

212 suggest that unhealthy ageing is mainly driven by an accelerated accumulation of

213 morbidities and by quadratic declines in function during old age. Second, they illustrate

214 how, despite having an increasing number of morbidities and functional problems over

215 time, mental health in older individuals seems to improve with age. Third, they show

216 that only individuals with very fast declines in function and fast accumulation of

217 morbidities present a significant worsening of self-rated health over time.

218 Consistent with extent findings, our analyses indicate that women [24] and participants

219 with low education [3;9] lose function and accumulate morbidities faster than their

220 counterparts. Moreover, our results extend knowledge about the timing of change in

221 slope of DA, and, as proposed by some authors [24], suggest that the health gap

222 between men and women may reach a plateau in the oldest age groups. Similarly, they

223 suggest that the observed differences across educational groups may level off with age,

224 which could be due to equalization of resources in later life and because of the selective 
survival of low-educated individuals who have acquired resistance against adversity $[25 ; 26]$. Still, we are aware that the ability to find statistical significant differences in DA slopes for the oldest age groups is limited by the relatively low number of subjects aged $>80$ in the present analyses.

Previous investigations have also reported improvements in mental health- and in particular of hedonic wellbeing- with advancing age [27]. This phenomenon has been attributed to increases in wisdom with age, so that, despite declining health, older people may experience less stress, anger or worry thanks to adaptive shifts in emotional regulation [28].

Our results are of clinical significance, as they show average annual increments in the overall DA index of 0.74 (0.69 to 0.79$)$ points, which are associated with a $1.02(95 \%$ : 1.01-1.03) increase risk of mortality in our cohort. In particular, men in our cohort suffer the highest risk of mortality associated with high DA index values (HR (95\%CI) for mortality among those in the second, third and fourth quartiles of the DA index compared to those in the lowest: $2.11(1.33,3.37), 2.63(1.58,4.37)$ and $2.79(1.66$ to 4.71) in men; $0.92(0.41,2.08), 1.21(0.51,2.84)$ and $2.54(1.18,5.47)$ in women). The pattern of sex differences in the frailty index and in mortality observed in our study is consistent with the so-called male-female health-survival paradox [29], which implies that women experience lower mortality than men despite their higher rates of DA. Future analyses should evaluate whether sex-differences in the type of deficits accumulated over time, could help explain this paradox [24].

Several limitations of the present study warrant consideration. First, even if analyses of the potential impact of attrition revealed no major differences in the main results, the fact that those who were lost to follow-up were more likely to be older, female and low 
educated might have led to underestimating the DA index slope. Another limitation is

250

251

252

253

254

255

256

257

258

259

260

261

262

263

264

265

266

267

268

269

270

271

that, despite our study included a greater number of health deficits than most previous reports $[3 ; 4 ; 6 ; 8]$, it did not include sensory impairments, which are highly prevalent and have shown to predict mortality in older adults [30]. Also, although adjustment for functionality was performed, results for dimensions that relied on self-reported data (i.e. the "self-rated health and vitality" dimension) may have been affected by the presence of cognitive impairment. Finally, our results cannot be extrapolated to institutionalized populations.

Still, our study has notable strengths compared to published research. First, it systematically evaluates for the first time the trajectories of DA by health domains, and addresses potential interactions between domains. Second, unlike most previous studies evaluating trajectories of DA based on self-reported data [3;4], ours included a number of validated measures of physical function (i.e. gait speed, grip strength) and chronic conditions (obesity, hypertension, diabetes), and adjusted for a range of potentially confounding health behaviors. Finally, our study expands the results of previous reports (mostly based on Anglo-Saxon cohorts) to a Mediterranean county, not only with one of the highest female life expectancies in the world [31], but also with very high rates of frailty [32].

\section{CONCLUSIONS}

Our results are of public health importance because they support that population-level interventions aimed to healthy ageing should focus on the prevention and control of chronic diseases, as well as on the preservation of function and early rehabilitation, with the latter being more relevant during the latter stages of life. The above implies that 
272 post-acute and long-term care services should incorporate adequate physical therapy and

273 nursing facilities to promote attainable levels of functionality in older adults.

274 
275 Funding: This work was mainly supported by FIS grant 16/609 (Instituto de Salud

276 Carlos III, State Secretary of R+D+I and FEDER/FSE) and grant no. 02/2014 from the

277 Plan Nacional sobre Drogas (Ministry of Health of Spain) and CIBERESP. Additional

278 funding was obtained from the ATHLOS project (EU H2020- Project ID: 635316) and

279 the Salamander Project (JPI-A Healthy Diet for a Healthy Life, State Secretary of

$280 \mathrm{R}+\mathrm{D}+\mathrm{I}$ PCIN-2016-145. The funding agencies had no role in study design, data

281 analysis, interpretation of results, manuscript preparation or in the decision to submit

282 this manuscript for publication.

283 Conflicts of interest: The authors declare that they have no conflicts of interest 


\section{REFERENCES}

(1) Lopez-Otin C, Blasco MA, Partridge L, Serrano M, Kroemer G. The hallmarks of aging. Cell 2013;153:1194-1217.

(2) Beard JR, Officer AM, Cassels AK. The World Report on Ageing and Health. Gerontologist 2016;56 Suppl 2:S163-S166.

(3) Yang Y, Lee LC. Dynamics and heterogeneity in the process of human frailty and aging: evidence from the U.S. older adult population. J Gerontol B Psychol Sci Soc Sci 2010;65B:246-255.

(4) Shi J, Yang Z, Song X et al. Sex differences in the limit to deficit accumulation in late middle-aged and older Chinese people: results from the Beijing Longitudinal Study of Aging. J Gerontol A Biol Sci Med Sci 2014;69:702-709.

(5) Rogers NT, Marshall A, Roberts CH, Demakakos P, Steptoe A, Scholes S. Physical activity and trajectories of frailty among older adults: Evidence from the English Longitudinal Study of Ageing. PLoS One 2017;12:e0170878.

(6) Marshall A, Nazroo J, Tampubolon G, Vanhoutte B. Cohort differences in the levels and trajectories of frailty among older people in England. J Epidemiol Community Health 2015;69:316-321.

(7) Lu W, Benson R, Glaser K et al. Relationship between employment histories and frailty trajectories in later life: evidence from the English Longitudinal Study of Ageing. J Epidemiol Community Health 2017;71:439-445.

(8) Chamberlain AM, Finney Rutten LJ, Manemann SM et al. Frailty Trajectories in an Elderly Population-Based Cohort. J Am Geriatr Soc 2016;64:285-292.

(9) Chamberlain AM, St Sauver JL, Jacobson DJ et al. Social and behavioural factors associated with frailty trajectories in a population-based cohort of older adults. BMJ Open 2016;6:e011410.

(10) Rockwood K. Conceptual Models of Frailty: Accumulation of Deficits. Can J Cardiol 2016;32:1046-1050.

(11) Rodriguez-Artalejo F, Graciani A, Guallar-Castillon P et al. [Rationale and methods of the study on nutrition and cardiovascular risk in Spain (ENRICA)]. Rev Esp Cardiol 2011;64:876-882.

(12) Garcia-Esquinas E, Andrade E, Martinez-Gomez D, Caballero FF, Lopez-Garcia $\mathrm{E}$, Rodriguez-Artalejo F. Television viewing time as a risk factor for frailty and functional limitations in older adults: results from 2 European prospective cohorts. Int J Behav Nutr Phys Act 2017;14:54.

(13) Pols MA, Peeters PH, Ocke MC, Slimani N, Bueno-de-Mesquita HB, Collette HJ. Estimation of reproducibility and relative validity of the questions included in the EPIC Physical Activity Questionnaire. Int J Epidemiol 1997;26 Suppl 1:S181-S189. 
(14) Martinez-Gonzalez MA, Lopez-Fontana C, Varo JJ, Sanchez-Villegas A, Martinez JA. Validation of the Spanish version of the physical activity questionnaire used in the Nurses' Health Study and the Health Professionals' Follow-up Study. Public Health Nutr 2005;8:920-927.

(15) Healy GN, Clark BK, Winkler EA, Gardiner PA, Brown WJ, Matthews CE. Measurement of adults' sedentary time in population-based studies. Am J Prev Med 2011;41:216-227.

(16) Kikuchi H, Inoue S, Sugiyama T et al. Distinct associations of different sedentary behaviors with health-related attributes among older adults. Prev Med 2014;67:335-339.

(17) Semanik PA, Lee J, Song J et al. Accelerometer-monitored sedentary behavior and observed physical function loss. Am J Public Health 2015;105:560-566.

(18) Andrade-Gomez E, Garcia-Esquinas E, Ortola R, Martinez-Gomez D, Rodriguez-Artalejo F. Watching TV has a distinct sociodemographic and lifestyle profile compared with other sedentary behaviors: A nationwide population-based study. PLoS One 2017;12:e0188836.

(19) Relative validity and reproducibility of a diet history questionnaire in Spain. I. Foods. EPIC Group of Spain. European Prospective Investigation into Cancer and Nutrition. Int J Epidemiol 1997;26 Suppl 1:S91-S99.

(20) Schroder H, Fito M, Estruch R et al. A short screener is valid for assessing Mediterranean diet adherence among older Spanish men and women. $J$ Nutr 2011;141:1140-1145.

(21) Raudenbush SW, Bryk AS. Hierarchichal Linear Models: Applications and Data Analysis Methods. 2nd edition ed. Thousand Oaks, CA: Sage Publications, 2002.

(22) Rabe-Hesketh S, Skrondal A. Generalized linear mixed-effects models. In: Fitzmaurice G, Davidian M, Verbeke G, Molenberghs G, eds. Longitudinal Data Analysis. Boca Raton, FL: Chapman and Hall/CRC; 2009;79-106.

(23) Greenland S. Dose-response and trend analysis in epidemiology: alternatives to categorical analysis. Epidemiology 1995;6:356-365.

(24) Gordon EH, Peel NM, Samanta M, Theou O, Howlett SE, Hubbard RE. Sex differences in frailty: A systematic review and meta-analysis. Exp Gerontol 2017;89:30-40.

(25) McMunn A, Nazroo J, Breeze E. Inequalities in health at older ages: a longitudinal investigation of the onset of illness and survival effects in England. Age Ageing 2009;38:181-187.

(26) Preston SH, Hill ME, Drevenstedt GL. Childhood conditions that predict survival to advanced ages among African-Americans. Soc Sci Med 1998;47:1231-1246. 
(27) Steptoe A, Deaton A, Stone AA. Subjective wellbeing, health, and ageing. Lancet 2015;385:640-648.

(28) Bangen KJ, Meeks TW, Jeste DV. Defining and assessing wisdom: a review of the literature. Am J Geriatr Psychiatry 2013;21:1254-1266.

(29) Alberts SC, Archie SC, Gesquiere LR, Altmann J, Vaupel JW, Christensen K. The Male-Female Health-Survival Paradox: A xomparative Persepective on Sex Differences in Aging and Mortality. In: Weinstein M, Lane MA, eds. Sociality, Hierarchy, Health: Comparative Biodemography. Washington (DC): National Academies Press (US); 2014.

(30) Pinto JM, Wroblewski KE, Huisingh-Scheetz M et al. Global Sensory Impairment Predicts Morbidity and Mortality in Older U.S. Adults. J Am Geriatr Soc 2017;65:2587-2595.

(31) United Nations DoEaSAPD. World Population Ageing 2015. ST/ESA/SER.A/390.

(32) Harttgen K, Kowal P, Strulik H, Chatterji S, Vollmer S. Patterns of frailty in older adults: comparing results from higher and lower income countries using the Survey of Health, Ageing and Retirement in Europe (SHARE) and the Study on Global AGEing and Adult Health (SAGE). PLoS One 2013;8:e75847. 
